\title{
Biomechanical Assessment of the Bone Ingrowth Effect During Cementless Endoprosthesis Osteointegration
}

\begin{abstract}
A. V. Nikitsin
Belarusian State University, Republic of Belarus, 220030, Minsk, Nezavisimosti avenue, 4, andy.nik @ tut.by

Finite element model of porous titanium inserts for cementless endoprosthesis was reconstructed using $X$-ray tomography. The stress distribution is calculated for a model with open-cell foam and composite bone / titanium. The results explain the mechanism of the porous structure destruction and positive influence of the osteointegration effect on the strength properties. Numerical calculations are confirmed by experimental data of the porous samples during compression testing. It is shown that changes in mechanical properties of porous titanium due to process of bone ingrowth is a topical area for biomechanical research.
\end{abstract}

Key words: FEA, stress-strain, osteointegration effect, $X$-ray tomography.

\section{References}

1. Shen H., Brinson L. C. Finite element modelling of porous titanium. International Journal of Solids and Structures, 2007, vol. 44, pp. 320-335.

2. Huanlong Li., Oppenheimer S. M., Stupp S. I., Dunand D. C., Brinston L. C. Effects of Pore Morphology and Bone Ingrowth on Mechanical Properties of Microporous Titanium as an Orthopaedic Implant Material. Materials Transactions, 2004, vol. 45, pp. 1124-1131.

3. Maslov A. P., Ruckij A. V., Nikitsin A. V. Perelomy bedrennogo komponenta jendoprotezov tazobedrennogo sustava sistemy SLPS [Fractures of the femoral component of the endoprosthesis hip joint system of SLPS]. Medicina, 2013, vol. 80, pp. 7-13. (in Russian) 4. Nikitsin A. V., Mikhasev G. I., Maslov A. P. Konechno-jelementnyj analiz vlijanija oblasti vrastanija na stabil'nost' jendoproteza tazobedrennogo sustava [Finite element analysis of the influence of the field ingrown on the stability of the hip joint endoprosthesis]. Mehanika mashin mehanizmov i materialov, 2012, vol. 18, pp. 8690. (in Russian).

5. Esen Z., Tarhan Bor E., Bor E. Characterization of loose powder sintered porous titanium and Ti6Al4V alloy. Turkish J.Eng.Env.Science, 2009, vol. 33, pp. 207-219.

6. Thelen S., Barthelat F., Brinson L.C. Mechanics considerations for micro-porous titanium as an orthopedic implant material. J. Biomed. Mater. Res. A, 2004, vol. 69, pp. 601-610.

7. Vasconcellos L., Leite D., Nascimento F., Graca M., Carvalho Y., Cairo C. . Porous titanium for biomedical applications: An experimental study on rabbits. Med. Oral. patol. Oral. Cir. Bucal., 2010, vol. 2, pp. 407-412.

8. Michailidis N., Stergioudi F., Omar H., Papadopoulos D., Tsipas D. N. Experimental and FEM analysis of the material response of porous metals imposed to mechanical loading. J. Colloids and Surfaces A: Physicochem. Eng. Aspects, 2011, vol. 382, pp. 124131.

9. Nikitsin A. V., Shil'ko S. V. Ocenka vlijanija osteogeneza na mehanicheskie svojstva poristogo titana pri szhatii [Estimation of influence of osteogenesis on mechanical properties porous titanium at compression]. Teoreticheskaja i prikladnaja mehanika, 2013, vol. 28, pp. 127-129. (in Russian).

10. Michailidis N., Stergioudi F., Omar H., Tsipas D. N. Investigation of the mechanical behaviour of open-cell $\mathrm{Ni}$ foams by experimental and FEM procedures. Advanced Engineering Materials, 2009, vol. 10, no. 12, pp. 11221126 .

\section{РОТАЦИОННАЯ ИНВАРИАНТНОСТЬ И ОБЪЕКТИВНЫЕ ФОРМЫ ЛАГРАНЖИАНОВ НЕЛИНЕЙНОГО МИКРОПОЛЯРНОГО ТЕРМОУПРУГОГО КОНТИНУУМА ВТОРОГО ТИПА}

\footnotetext{
Ю. Н. Радаев ${ }^{1}$, В. А. Ковалев ${ }^{2}$

${ }^{1}$ Доктор фризико-математических наук, просрессор, ведущий научный сотрудник, Институт проблем механики им. А. Ю. Ишлинского РАН, Москва, radayev@ipmnet.ru, y.radayev@gmail.com

${ }^{2}$ Доктор фризико-математических наук, зав. касредрой прикладной математики и аналитической поддержки принятия решений, Московский городской университет управления Правительства Москвы, kovalev.kam@gmail.com

В работе излагаются новые результаты в области распространения лагранжева полевого формализма на нелинейные связанные микрополярные термоупругие среды. Рассматривается теоретико-полевая модель микрополярного (МР) термоупругого (TE) континуума второго типа (GNII). Приводятся фрормулировка принципа наименьшего термоупругого действия и вывод дифрференциальных уравнений связанного микрополярного термоупругого поля в фрорме Эйлера-Лагранжа (EL). Обсуждаются вопросы, касающиеся инвариантности интегрального срункционала действия относительно сдвигов эйлеро-
} 
вых полевых переменных, времени и температурного смещения, а также трехмерных вращений эйлеровой координатной системы. Исследуется проблема ротационной инвариантности «естественной» плотности микрополярного термоупругого действия. Получены срункциональные условия ротационной инвариантности действия, независимые ротационно инвариантные аргументы и удовлетворяющая принципу объективности фрорма свободной энергии Гельмгольца.

Ключевые слова: микрополярная термоупругость второго типа, поле, экстраполе, действие, лагранжиан, $d$-вектор, инвариантность, поворот, принцип объективности, десрормация, экстрадесрормация.

\section{1. ДЕФОРМАЦИЯ И ЭКСТРАДЕФОРМАЦИЯ МИКРОПОЛЯРНОГО КОНТИНУУМА. ПОЛЯРНЫЕ $d$-ВЕКТОРЫ}

В математических теориях континуума с полярной микроструктурой (см., например, $[1,2]$ ) предполагается, что произвольная «конечная» деформация континуума, представляемая чисто геометрическим преобразованием

$$
\mathbf{x}=\mathbf{x}(\mathbf{X}, t)
$$

положения $\mathbf{X}$ отсчетной конфигурации в соответствующее актуальное место $\mathbf{x}$ пространства, сопровождается экстрадеформацией, проявляющейся в форме нарушений взаимной ориентации и метрических характеристик системы трех некомпланарных $d$-векторов $\underset{\mathfrak{a}}{\mathbf{d}}(\mathfrak{a}=1,2,3)$, связанных с микроэлементом:

$$
\underset{\mathfrak{a}}{\mathbf{d}}=\underset{\mathfrak{a}}{\mathbf{d}}(\mathbf{X}, t) .
$$

Система трех пространственных полярных $d$-векторов, ассоциированных с каждой точкой континуума, задает микрополярную структуру континуума. Эта система в самом общем случае предполагается «мягкой».

Переменные $\mathbf{X}$ и $\mathbf{x}$ (и позиционные координаты $X^{\alpha}, x^{j}$ ) выступают как соответственно лагранжева (отсчетная, референциальная) и эйлерова (пространственная) переменные, если воспользоваться стандартной терминологиней механики континуума $[3,4]$. С этими переменными связаны метрики: отсчетная метрика ' $g_{\alpha \beta}$ и пространственная метрика $g_{i j}$. Конвективная (сопутствующая) метрика характеризуется метрическим тензором $g_{\alpha \beta}$ и, в отличии от ' $g_{\alpha \beta}$ и $g_{i j}$, определяется деформацией (1). Как ясно из предложенных обозначений, эйлеровы пространственные индексы всегда будут обозначаться латинскими буквами, греческие буквы всегда будут указывать на отсчетные или сопутствующие индексы.

Деформация и экстрадеформация в координатах $X^{\alpha}, x^{j}$ имеют следующий вид:

$$
\begin{aligned}
& x^{j}=x^{j}\left(X^{\alpha}, t\right), \\
& d_{\mathfrak{a}}^{j}=d_{\mathfrak{a}}^{j}\left(X^{\alpha}, t\right) .
\end{aligned}
$$

Следуя известным схемам построения математических теорий континуумов, введем градиент «конечной» деформации (градиент места, position gradient), или «дисторсию» $[5,6]$ :

$$
\partial_{\alpha} x^{j} \quad(j, \alpha=1,2,3)
$$

и соответствующий якобиан

$$
J=\operatorname{det}\left(\partial_{\alpha} x^{j}\right) .
$$

Конвективная метрика вычисляется с помощью градиента деформации согласно формуле

$$
g_{\alpha \beta}=g_{i j}\left(\partial_{\alpha} x^{i}\right)\left(\partial_{\beta} x^{j}\right)
$$

и в силу своего определения ротационно инвариантна при произвольных поворотах эйлеровой координатной системы.

Заметим, что в рамках теоретико-полевого подхода лагранжевы переменные $X^{\alpha}(\alpha=1,2,3)$, дополненные четвертой временной координатой, выступают как пространственно-временные координаты. Эйлеровы переменные $x^{j}(j=1,2,3)$ представляют собой физические поля. То же самое относится к «мягкой» системе полярных $d$-векторов $\underset{\mathfrak{a}}{\mathbf{d}}(\mathfrak{a}=1,2,3)$. Но они классифицируются в дальнейшем как 
экстраполевые (сверхполевых переменных $x^{j}$ ) переменные и вводятся в формализм теории поля с помощью контравариантных пространственных компонент $d^{j}(\mathfrak{a}=1,2,3 ; j=1,2,3)$.

В качестве основной термической полевой переменной, следуя теориям термоупругости второго типа, примем температурное смещение $\vartheta$, которое определяется как первообразная по времени (при фиксированных лагранжевых переменных) от абсолютной температуры $\theta$. Переменной состояния считается также референциальный градиент температурного смещения (так называемая «быстрая» переменная термодинамического состояния).

Таким образом, определяющие полевые переменные микрополярного термоупругого континуума второго типа, помимо $x^{j}(j=1,2,3)$ и $\vartheta$, включают:

- градиент деформации $\partial_{\alpha} x^{j}(j, \alpha=1,2,3)$;

- три пространственных $d$-вектора «нежесткой» микрополярной структуры континуума $d_{\mathfrak{a}}^{j}$ $(\mathfrak{a}=1,2,3 ; j=1,2,3) ;$

- референциальныые градиенты $d$-векторов $\partial_{\alpha} d_{\mathfrak{a}}^{j}(\mathfrak{a}=1, \mid 2,3 ; j=1,2,3 ; \alpha=1,2,3)$;

- референциальный градиент температурного смещения $\partial_{\alpha} \vartheta(\alpha=1,2,3)$;

- скорость температурного смещения $\partial_{4} \vartheta$.

\section{2. СВЯЗАННЫЙ МИКРОПОЛЯРНЫЙ ТЕРМОУПРУГИЙ КОНТИНУУМ ВТОРОГО ТИПА. ДЕЙСТВИЕ. ЛАГРАНЖИАН. ДИФФЕРЕНЦИАЛЬНЫЕ УРАВНЕНИЯ ПОЛЯ}

Теоретико-полевая модель подразумевает задание интегрального функционала действия и соответствующей плотности действия. В дальнейшем мы будем использовать «естественные» плотности (см., например, [7]), называемые еще «квазиплотностями». «Естественная» плотность действия (лагранжиан) микрополярного термоупругого континуума может быть, как это принято в теориях термоупругости второго типа [7], представлена в виде функции с явными вхождениями определяющих переменных:

$$
\mathscr{L}=\mathscr{L}\left(X^{\beta}, x^{j}, d_{\mathfrak{a}}^{j}, \vartheta, \dot{x}^{j}, \dot{d}_{\mathfrak{a}}^{j}, \dot{\vartheta}, \partial_{\alpha} x^{j}, \partial_{\alpha} d_{\mathfrak{a}}^{j}, \partial_{\alpha} \vartheta\right)
$$

В теориях континуумов лагранжиан имеет несколько более специальную форму, чем (8), разности плотности кинетической энергии и свободной энергии Гельмгольца:

$$
\mathscr{L}=\frac{1}{2} \rho_{\mathrm{R}} g_{k j} \dot{x}^{k} \dot{x}^{j}+\frac{1}{2} \rho_{\mathrm{R}} g_{i j} \underset{\mathfrak{I} \mathfrak{\mathfrak { I }}}{\stackrel{\mathfrak{a}}{d_{\mathfrak{a}}} \dot{d}^{i}} \dot{d}^{j}-\psi\left(X^{\beta}, x^{j}, d_{\mathfrak{a}}^{j}, \vartheta, \dot{x}^{j}, \dot{d}_{\mathfrak{a}}^{j}, \dot{\vartheta}, \partial_{\alpha} x^{j}, \partial_{\alpha} d_{\mathfrak{a}}^{j}, \partial_{\alpha} \vartheta\right),
$$

где точкой (вместо оператора $\partial_{4}$ ) обозначается частное дифференцирование по времени при постоянных лагранжевых координатах $X^{\alpha}(\alpha=1,2,3) ; \rho_{\mathrm{R}}-$ референциальная плотность; $\mathfrak{J}-$ тензор инерции микроэлемента. Явная зависимость лагранжиана $\mathscr{L}$ от времени исключается нами а priori.

Вариационный интеграл термоупругого действия с «естественной» плотностью действия (8) будет иметь следующий вид:

$\operatorname{Im}=\int \mathscr{L}\left(X^{\beta}, x^{j}, d_{\mathfrak{a}}^{j}, \vartheta, \dot{x}^{j}, \dot{d}_{\mathfrak{a}}^{j}, \dot{\vartheta}, \partial_{\alpha} x^{j}, \partial_{\alpha} d_{\mathfrak{a}}^{j}, \partial_{\alpha} \vartheta\right) d^{4} X \quad(\mathfrak{a}=1,2,3 ; \alpha, \beta=1,2,3 ; j=1,2,3)$.

Соответствующие вариационному интегралу (10) и принципу наименьшего действия связанные уравнения поля могут быть представлены в стандартной форме Эйлера-Лагранжа (EL):

$$
\begin{aligned}
& \partial_{\alpha} S_{\cdot j}^{\alpha \cdot}-\dot{P}_{j}=-\frac{\partial \mathscr{L}}{\partial x^{j}} \quad(\alpha=1,2,3 ; j=1,2,3), \\
& \partial_{\alpha} \stackrel{\mathfrak{a}}{\mathscr{M}} \cdot \cdot j^{\alpha \cdot j}+\stackrel{\mathfrak{a}}{\mathscr{A}}_{j}-\partial_{4} \stackrel{\mathfrak{a}}{\mathscr{Q}}_{j}=0 \quad(\mathfrak{a}=1,2,3 ; \alpha=1,2,3 ; j=1,2,3), \\
& \partial_{\alpha} j_{\mathrm{R}}^{\alpha}+\dot{s}=\frac{\partial \mathscr{L}}{\partial \vartheta} \quad(\alpha=1,2,3) \text {. }
\end{aligned}
$$

Здесь принимаются следующие обозначения для полевых частных производных, которые вводятся с целью дать по возможности более компактную запись дифференциальных уравнений поля (11):

$$
P_{j}=\frac{\partial \mathscr{L}}{\partial \dot{x}^{j}}, \quad \stackrel{\mathfrak{Q}}{\mathcal{Q}}_{j}=\frac{\partial \mathscr{L}}{\partial \dot{d}_{\mathfrak{a}}^{j}},
$$




$$
\begin{gathered}
S_{\cdot j}^{\alpha \cdot}=-\frac{\partial \mathscr{L}}{\partial\left(\partial_{\alpha} x^{j}\right)}, \quad \mathscr{M}_{\cdot j}^{\alpha \cdot}=-\frac{\partial \mathscr{L}}{\partial\left(\partial_{\alpha} d^{j}\right)} \\
\mathscr{A}_{j}=\frac{\partial \mathscr{L}}{\partial d_{\mathfrak{a}}^{j}}, \quad s=\frac{\partial \mathscr{L}}{\partial \dot{\vartheta}}, \quad j_{\mathrm{R}}^{\alpha}=\frac{\partial \mathscr{L}}{\partial\left(\partial_{\alpha} \vartheta\right)}
\end{gathered}
$$

Поясним смысл символов, использованных в данных выше формулах (12) с целью сокращенного обозначения полевых частных производных:

$P_{j}$ - обобщенный импульс, соответствующий трансляционным степеням свободы;

$\stackrel{\mathfrak{a}}{\mathscr{Q}}_{j}$ - обобщенные экстраимпульсы, соответствующие дополнительным степеням свободы;

$S_{\cdot j}^{\alpha \cdot}-$ первый тензор напряжений Пиола-Кирхгофа;

$\mathscr{\mathscr { M }}_{\cdot j}^{\alpha \cdot}-$ «первые» тензоры экстранапряжений;

$\mathscr{A}_{j}$ - обобщенные силы-моменты, сопряженные экстраполевым переменным $d^{j}(\mathfrak{a}=1,2,3$; $j=1,2,3)$;

$s-$ плотность энтропии (в расчете на единицу объема в отсчетном состоянии);

$j_{\mathrm{R}}^{\alpha}-$ референциальный вектор потока энтропии (в единицу времени через единицу площади в отсчетном состоянии).

\section{3. РОТАЦИОННАЯ ИНВАРИАНТНОСТЬ ДЕЙСТВИЯ И ПЛОТНОСТИ ДЕЙСТВИЯ ОТНОСИТЕЛЬНО ПОВОРОТОВ ЭЙЛЕРОВОЙ КООРДИНАТНОЙ СИСТЕМЫ}

«Естественная» плотность действия в форме (8) пока еще не позволяет вести речь о ее объективности в том смысле, что в разных эйлеровых координатных системах эта форма будет сохраняться. Ясно, что вывод объективных форм лагранжиана представляет собой первый и весьма важный шаг на пути построения объективных форм определяющих уравнений, первоначально задаваемых уравнениями (12).

Для изображения состояний и процессов в механике континуума используется трехмерное плоское пространство-время и независимое время. Поскольку выбор эйлеровых координат произволен и не должен никак сказываться на физических следствиях дифференциальных уравнений поля, то действие и лагранжиан обязаны обладать определенными свойствами инвариантности по отношению к выбору эйлеровой координатной системы и начала отсчета времени, т. е. по отношению к так называемым «движениям» эйлерова пространства. Существуют два принципиально различных вида «движений»: трансляционные и спинорные. Первые определяются заданием векторов положений и описывают перемещения (трансляции) тел в эйлеровом пространстве. Спинорные «движения»определяются заданием тензорных функций времени, значениями которых являются собственно ортогональные тензоры размерности три (тензоры поворота).

Вводя в пространстве прямоугольные декартовы координаты $x^{j}$, заметим, что одно из таких свойств инвариантности проявляется в форме трансляционной инвариантности интегрального функционала действия (10) относительно произвольных сдвигов переменных $x^{j}$ и времени $t$. Другое, как хорошо известно, - ротационной инвариантности относительно произвольных поворотов эйлеровой координатной системы $x^{j}$.

Инвариантность действия относительно поворотов эйлерова координатного репера является проявлением изотропии эйлерова координатного пространства, т. е. отсутствия предпочтительных направлений в этом пространстве.

Инвариантность действия относительно преобразований лагранжевых переменных связана с симметрией физических свойств континуума. Так, трансляционная инвариантность действия (10) относительно произвольных сдвигов координат $X^{\alpha}$ означает, что континуум однороден. Ротационная инвариантность относительно произвольных поворотов лагранжевой координатной системы указывает на изотропность континуума.

Таким образом, действие, в частности, должно быть инвариантно относительно преобразований сдвигов и поворотов координатной системы наблюдателя (принцип объективности) и сдвигов времени:

$$
\widetilde{x}^{i}=R_{j}^{i} x^{j}+C^{i}, \quad \widetilde{d}_{\mathfrak{a}}^{i}=R_{j}^{i} d_{\mathfrak{a}}^{j}, \quad \tilde{t}=t+C .
$$


В приведенных выше формулах преобразования $C^{i}, C$ есть произвольные постоянные; $R_{j}^{i}-$ произвольная постоянная собственно ортогональная матрица.

Действие (10) и плотность действия $\mathscr{L}$ инвариантны относительно преобразований (13) тогда и только тогда, когда выполнены следующие условия:

$$
\frac{\partial \mathscr{L}}{\partial x^{k}}=0, \quad \partial_{4}^{\operatorname{expl}} \mathscr{L}=0, \quad \mathscr{K}_{[i j]}=0,
$$

где тензор $\mathscr{K}_{i j}$ определяется согласно

$$
\mathscr{K}_{i j}=x_{i} \frac{\partial \mathscr{L}}{\partial x^{j}}+\underset{\mathfrak{a}}{d_{i}} \frac{\partial \mathscr{L}}{\partial d_{\mathfrak{a}}^{j}}+\dot{x}_{i} \frac{\partial \mathscr{L}}{\partial \dot{x}^{j}}+\dot{d}_{\mathfrak{a}} \frac{\partial \mathscr{L}}{\partial \dot{d}_{\mathfrak{a}}^{j}}+\left(\partial_{\alpha} x_{i}\right) \frac{\partial \mathscr{L}}{\partial\left(\partial_{\alpha} x^{j}\right)}+\left(\partial_{\alpha} d_{\mathfrak{a}}\right) \frac{\partial \mathscr{L}}{\partial\left(\partial_{\alpha} d_{\mathfrak{a}}^{j}\right)}
$$

и в (14) по индесам, заключенным в квадратные скобки, выполняется антисимметризация.

Заметим, что в силу (14) и в обозначениях (12) тензор $\mathscr{K}_{i j}$ сводится к

$$
\mathscr{K}_{i j}=\underset{\mathfrak{a}}{d_{i} \mathscr{A}_{j}}+\dot{x}_{i} P_{j}+\dot{d}_{\mathfrak{a}} \stackrel{\mathfrak{Q}}{j}_{j}-\left(\partial_{\alpha} x_{i}\right) S_{\cdot j}^{\alpha \cdot}-\left(\partial_{\alpha} d_{\mathfrak{a}}\right) \mathscr{M}_{\cdot j}^{\alpha} .
$$

Ясно, что в том случае, когда плотность действия не зависит явно от директоров $d_{\mathfrak{a}}^{j}$, их производных по времени $\underset{\mathfrak{a}}{\dot{d}}$ и референциальных градиентов $\partial_{\alpha} d_{\mathfrak{a}}^{j}$, последнее в группе условий (14) позволяет сразу же установить симметрию тензора напряжений Коши:

$$
T_{\cdot k}^{l \cdot}=-J^{-1}\left(\partial_{\beta} x^{l}\right) \frac{\partial \mathscr{L}}{\partial\left(\partial_{\beta} x^{k}\right)} \quad(\beta=1,2,3) .
$$

Инвариантность действия относительно трансляций эйлеровых координат, известная как принцип галилеевой инвариантности действия (принцип относительности Галилея), мы дополним требованием инвариантности действия относительно сдвигов температурного смещения $\left(C^{\prime}-\right.$ произвольная постоянная):

$$
\widetilde{\vartheta}=\vartheta+C^{\prime}
$$

что обеспечивается выполнением следующего условия:

$$
\frac{\partial \mathscr{L}}{\partial \vartheta}=0 .
$$

Поскольку кинетическая составляющая плотности действия инвариантна относительно преобразований (13), (18), то плотность свободной энергии Гельмгольца $(\alpha, \beta=1,2,3)$

$$
\psi=\psi\left(X^{\beta}, d_{\mathfrak{a}}^{j}, \dot{\vartheta}, \partial_{\alpha} x^{j}, \partial_{\alpha} d_{\mathfrak{a}}^{j}, \partial_{\alpha} \vartheta\right),
$$

в свою очередь, обязана выдерживать преобразования вида (13), (18), т. е.

$$
\psi\left(X^{\beta}, R_{j}^{i} d_{\mathfrak{a}}^{j}, \dot{\vartheta}, R_{j}^{i} \partial_{\alpha} x^{j}, R_{j}^{i} \partial_{\alpha} d_{\mathfrak{a}}^{j}, \partial_{\alpha} \vartheta\right)=\psi\left(X^{\beta}, \underset{\mathfrak{a}}{d^{j}}, \dot{\vartheta}, \partial_{\alpha} x^{j}, \partial_{\alpha} d_{\mathfrak{a}}^{j}, \partial_{\alpha} \vartheta\right) .
$$

Последнее обстоятельство означает, что свободная энергия Гельмгольца является некоторой функцией от переменных

$$
X^{\beta}, \quad \dot{\vartheta}, \quad \partial_{\alpha} \vartheta,
$$

в запись которых не входят эйлеровы индексы, а также следующих инвариантных относительно вращений эйлеровой координатной системы аргументов:

$$
\begin{gathered}
g_{\alpha \beta}=g_{i j}\left(\partial_{\alpha} x^{i}\right)\left(\partial_{\beta} x^{j}\right), \\
\mathscr{R}_{\alpha}=g_{i j}\left(\partial_{\alpha} x^{i}\right) d_{\mathfrak{a}}^{j}, \\
\underset{\mathfrak{a}}{\mathscr{T}} \alpha \beta=g_{i j}\left(\partial_{\alpha} x^{i}\right)\left(\underset{\beta}{\partial_{\mathfrak{a}} d^{j}}\right) .
\end{gathered}
$$


Каждая из величин, перечисленных в (22), действительно инвариантна относительно произвольных вращений эйлеровой координатной системы, поскольку по всем эйлеровым индексам производится сворачивание с помощью эйлеровых метрических коэффициентов $g_{i j}$.

Заметим, что в списке инвариантных аргументов (22) отсутствуют тензоры

$$
\begin{gathered}
\mathscr{R}=g_{i j} d_{\mathfrak{a}}^{i} d^{j}, \\
\underset{\mathfrak{a} \mathfrak{b}}{\mathscr{R}}, g_{i j}\left(\partial_{\alpha} d_{\mathfrak{a}}^{i}\right) d_{\mathfrak{b}}^{j}, \\
\underset{\mathfrak{a} \mathfrak{b}}{\mathscr{T}} \alpha \beta=g_{i j}\left(\partial_{\alpha} d_{\mathfrak{a}}^{i}\right)\left(\partial_{\mathfrak{b}} d^{j}\right) .
\end{gathered}
$$

Рациональной основой для этого выступает требование того, чтобы экстрадеформация континуума была невозможна, если отсутствует деформация $\left(g_{\alpha \beta}={ }^{\prime} g_{\alpha \beta}\right)$.

Заметим также, что кинематическое ограничение

$$
\underset{\mathfrak{a} \mathfrak{b}}{\mathscr{R}}=\underset{\mathfrak{a} \mathfrak{b}}{\mathfrak{b}}
$$

устанавливает, что $d$-векторы составляют «жесткий» репер, поэтому экстрадеформация континуума сводится лишь к вращениям составляющих его элементов.

В итоге, считая, что континуум однороден, т. е.

$$
\partial_{\beta}^{\operatorname{expl}} \mathscr{L}=0 \quad(\beta=1,2,3),
$$

и, следовательно, все лагранжевы переменные $X^{\beta}$ являются циклическими (игнорируемыми), получаем следующую, удовлетворяющую принципу объективности, ротационно-инвариантную форму свободной энергии Гельмгольца $(\mathfrak{a}=1,2,3 ; \alpha, \beta=1,2,3)$ :

$$
\psi=\psi\left(g_{\alpha \beta}, \underset{\mathfrak{a}}{\alpha}, \underset{\mathfrak{a}}{\alpha \beta}, \dot{\vartheta}, \partial_{\alpha} \vartheta\right)
$$

Мы неявно подразумеваем, что приведенная форма (25) должна зависеть также от отсчетной метрики ' $g_{\alpha \beta}$ и референциального положения $d$-векторов ' $d_{\mathfrak{a}}^{j}(\mathfrak{a}=1,2,3)$.

В форме (25) ротационно-инвариантный аргумент $g_{\alpha \beta}$ без ограничения общности может быть заменен на

$$
\epsilon_{\alpha \beta}=\frac{1}{2}\left(g_{\alpha \beta}-{ }^{\prime} g_{\alpha \beta}\right) \text {. }
$$

Компоненты $\epsilon_{\alpha \beta}$ преобразуются по тензорному закону при заменах лагранжевых координат. Тензор $\epsilon_{\alpha \beta}$ называется тензором деформации Грина. Использование тензора деформации Грина в качестве ротационно инвариантного аргумента лагранжиана исключительно удобно, так как он в силу своего определения учитывает только ту часть деформации континуума, которая наблюдается относительно некоторой фиксированной референциальной конфигурации.

По аналогичным соображениям вместо векторной меры экстрадеформации $\underset{\mathfrak{a}}{\alpha}$ следует использовать относительный вектор экстрадеформации

$$
-\gamma_{\mathfrak{a}}=\underset{\mathfrak{a}}{\mathscr{R}} \alpha \underset{\alpha \beta}{\stackrel{1}{\prime} d^{\beta}}
$$

Здесь векторы ' ${ }_{\mathfrak{a}}^{\beta}$ указывают референциальное состояние системы $d$-векторов. Вектор $\gamma_{\mathfrak{a}}$ оказывается нулевым, только если каждый из $d$-векторов поворачивается и удлиняется так, как это в точности предписывается деформацией континуума. Если последнее обстоятельство имеет место, то

$$
d_{\mathfrak{a}}^{i}-\left(\partial_{\alpha} x^{i}\right)^{1} d_{\mathfrak{a}}^{\alpha}=0
$$

умножая обе части полученного равенства на компоненты дисторсии $\partial_{\beta} x^{j}$ и сворачивая с $g_{i j}$, находим

$$
\underset{\mathfrak{a}}{\mathscr{R}}-g_{\beta \alpha}{ }^{\prime} d_{\mathfrak{a}}^{\alpha}=0
$$


т. е. относительный вектор экстрадеформации становится равным нулю:

$$
\gamma_{\beta}=0
$$

Таким образом, окончательно ротационно инвариантная форма свободной энергии Гельмгольца получается в виде

$$
\psi=\psi\left(\epsilon_{\alpha \beta}, \gamma_{\mathfrak{a}}, \mathscr{T}_{\mathfrak{a}} \alpha, \dot{\vartheta}, \partial_{\alpha} \vartheta\right) \quad(\mathfrak{a}=1,2,3 ; \alpha, \beta=1,2,3)
$$

Работа выполнена при частичной финансовой поддержке Российского фонда фундаментальных исследований (проект 13-01-00139 «Гиперболические тепловые волны в твердых телах с микроструктурой»).

\title{
Библиографрический список
}

1. Cosserat E. et $F$. Théorie des corps déformables. Paris : Librairie Scientifique A. Hermann et Fils, 1909. 226 p.

2. Toupin R. A. Theories of Elasticity with Couplestress // Arch. Rational Mech. Anal. 1964. Vol. 17, № 5. P. 85-112.

3. Седов Л. И. Введение в механику сплошных сред. М. : Физматгиз, 1962. 284 с.

4. Ильюшин А. А. Механика сплошных сред. М. : Издво Моск. ун-та, 1978. 287 с.

5. Грин А., Адкинс Дж. Большие упругие деформации и нелинейная механика сплошной среды. М. : Мир, 1965. $456 \mathrm{c}$.

6. Бердичевский В. Л. Вариационные принципы механики сплошной среды. М. : Наука, 1983. 448 с.

7. Ковалев В. А., Радаев Ю. Н. Волновые задачи теории поля и термомеханика. Саратов : Изд-во Сарат. ун-та, 2010. 328 c.

\section{Rotational Invariance of Non-Linear Lagrangians of Type-II Micropolar Thermoelastic Continuum}

\begin{abstract}
Y. N. Radayev' ${ }^{1}$, V. A. Kovalev ${ }^{2}$
${ }^{1}$ Institute for Problems in Mechanics of the Russian Academy of Sciences, Russia, 119526, Moscow, pr. Vernadskogo, 101, block 1, radayev@ipmnet.ru, y.radayev@gmail.com

${ }^{2}$ Moscow City Government University of Management Moscow, Russia, 107045, Moscow, Sretenka st., 28, kovalev.kam @ gmail.com

The paper contains new results related to extension of the field theoretical approach and its formalism to non-linear coupled micropolar thermoelastic media. A mathematical model of micropolar (MP) type-II (GNII) thermoelastic (TE) continuum is considered. A formulation of the least thermoelastic action principle is discussed. Partial differential equations subsequent to the least action principle are derived. The translational symmetries of non-linear Lagrangians are adopted. Those include an additional symmetry: translations of the thermal displacement. The rotational invariance of the action and corresponding Lagrangian is then studied. For micropolar type-II thermoelastic Lagrangians following the usual procedure independent rotationally invariant functional arguments are obtained. Objective forms of the Lagrangians satisfying the frame indifference principle are given.

Key words: type-II micropolar thermoelasticity, field, extra field, action, Lagrangian, $d$-vector, invariance, rotation, frame indifference principle, deformation, extra deformation.
\end{abstract}

\section{References}

1. Cosserat E. et F. Théorie des corps déformables. Paris, Librairie Scientifique A. Hermann et Fils, 1909, 226 p.

2. Toupin R. A. Theories of Elasticity with Couple-stress. Arch. Rational Mech. Anal. 1964, vol. 17, no. 5, pp. 85112.

3. Sedov L. I. Vvedenie v mekhaniku sploshnykh sred [Introduction to Mechanics of Continuos Media]. Moscow, Fizmatgiz, 1962, 284 p. (in Russian).

4. Illyushin A. A. Mekhanika sploshnykh sred [Mechanics of Continuos Media]. Moscow, Moscow Univ. Press., 1978, 287 p. (in Russian).
5. Green A. E., Adkins J. E. Bol'shie uprugie deformatsii $i$ nelineinaia mekhanika sploshnoi sredy [Large Elastic Deformations and Non-Linear Continuum Mechanics]. Moscow, Mir, 1965, 456 p. (in Russian).

6. Berdichevskii V. L. Variatsionnye printsipy mekhaniki sploshnoi sredy [Variational Principles of Mechanics of Continua]. Moscow, Nauka, 1983, 448 p. (in Russian). 7. Kovalev V. A., Radayev Y. N. Volnovye zadachi teorii polia i termomekhanika [Wave Problems of Field Theory and Thermomechanics]. Saratov. Saratov Univ. Press., 2010, 328 p. (in Russian). 